\title{
FITOSSOCIOLOGIA EM FRAGMENTO FLORESTAL NO NOROESTE DO ESTADO DO RIO GRANDE DO SUL
}

\author{
PHYTOSOCIOLOGICAL STUDY IN A FOREST FRAGMENT IN THE \\ NORTHWEST OF RIO GRANDE DO SUL STATE
}

\author{
Marcelo Callegari Scipioni ${ }^{1}$ César Augusto Guimarães Finger ${ }^{2}$ \\ Edison Bisognin Cantarelli ${ }^{3}$ Luciano Denardi ${ }^{3}$ Evandro Alcir Meyer ${ }^{4}$
}

\begin{abstract}
RESUMO
O presente estudo foi realizado em Frederico Westphalen, no Estado do Rio Grande do Sul, com a instalação de 10 parcelas de 20x50 m. Em cada parcela foram levantadas todas as plantas com diâmetro a altura do peito (DAP) $\geq 5 \mathrm{~cm}$, totalizando 1.461 indivíduos amostrados no hectare. A florística da área inclui 79 espécies distribuídas em 31 famílias e 69 gêneros. A família Fabaceae, com 14 espécies, foi a mais diversa, seguido por Myrtaceae (8), Meliaceae (6), Euphorbiaceae (5), Sapindaceae (5), Rutaceae (4) e Salicaceae (4). As espécies com maior valor de importância (VI) foram Gymnanthes concolor, Nectandra megapotamica, Trichilia claussenii e Chrysophyllum marginatum. Os índices de diversidade de Shannon e equabilidade de Pielou foram respectivamente 3,49 nats. ind..$^{-1}$ e 0,79 . Na análise de agrupamento de Twinspan foi contatado pela florística três subformações no fragmento, e destas, uma foi fisionomicamente distinta pelo estrato inferior, com o predomínio de espécies arborescentes (xaxim), enquanto as demais foram caracterizadas por espécies arbóreas, sendo as espécies, Trichilia claussenii e Gymnanthes concolor, as responsáveis pelo grande grupo fisionômico, que caracterizou a sucessão florestal em secundária avançada.
\end{abstract}

Palavras-chave: grupos ecológicos; floresta secundária; sucessão florestal; pteridófita arborescente.

\begin{abstract}
For the current study, 10 plots measuring 20x50 meters were created in the city of Frederico Westphalen, in the state of Rio Grande do Sul, Brazil. All plants within these plots with a diameter at breast height (DBH) of $\geq 5$ $\mathrm{cm}$ were identified, resulting in a total of 1,461 plants per hectare. The floristic composition revealed 79 species belonging to 31 families and 69 genera. The Fabaceae was the richest family in this study, having 14 species. Other families which presented a great number of species were the Myrtaceae (8), Meliaceae (6), Euphorbiaceae (5), Sapindaceae (5), Rutaceae (4) and Salicaceae (4). The species with the greatest importance values (IV) were the Gymnanthes concolor, Nectandra megapotamica, Trichilia claussenii and Chrysophyllum marginatum. The Shannon Diversity index and the Pielou Equability index were 3.49 nats. ind.$^{-1}$ and 0.79 , respectively. A Twinspan classification analysis indicated three floristic subgroups in the forest patch. From these three groups, one was physionomically distinct because of its lower canopy and the predominance of tree fern species. The other two ones were characterized by tree species such as the Trichilia claussenii and Gymnanthes concolor, which are responsible for the large physiognomic group that characterized advanced secondary succession forests.
\end{abstract}

Keywords: ecological groups; secondary forest; forest succession; tree fern.

1. Engenheiro Agrônomo e Florestal, MSc., Doutorando no Programa de Pós-Graduação em Engenharia Florestal, Centro de Ciências Florestais e da Madeira, Setor de Ciências Agrárias, Universidade Federal do Paraná, CEP 80210-170, Curitiba (PR). marcelo.scipioni@gmail.com

2. Engenheiro Florestal, Dr., Professor do Departamento de Ciências Florestais, Centro de Ciências Rurais, Universidade Federal de Santa Maria, Av. Roraima, 1000, CEP 97105-900, Santa Maria (RS). finger@smail.ufsm.br

3. Engenheiro Florestal, Dr., Professor do Departamento de Engenharia Florestal, Centro de Educação Superior Norte do Rio Grande do Sul, Universidade Federal de Santa Maria, Linha 7 de Setembro s/n, BR386 Km 40, CEP 98400-000, Frederico Westphalen (RS). ecantarelli@smail.ufsm.br/lucianodenardi@yahoo.com.br

4. Engenheiro Florestal, Mestrando no Programa de Pós-Graduação em Engenharia Florestal, Centro de Ciências Rurais, UFSM, Av. Roraima, 1000, CEP 97105-900, Santa Maria (RS).meyerfloresta@yahoo.com.br

Recebido para publicação em 14/08/2009 e aceito em 2/09/2010 


\section{INTRODUÇÃO}

As florestas estacionais são as formações florestais com maior área de cobertura entre as ocorrentes no Rio Grande do Sul (RS), predominando no Alto Uruguai, ao longo das encostas da Serra Geral e leste do Planalto Sul-Rio-Grandense ou Serra do Sudeste (RAMBO, 1961).

Ao longo do Rio Uruguai e seus afluentes, se estende uma densa floresta, caracterizada por um estrato arbóreo superior formado por árvores altas e emergentes, na sua maioria decidual. Nesse caso, dominam principalmente a grápia (Apuleia leiocarpa), sem dúvida a árvore mais frequente ao longo das encostas do Rio Uruguai, o angicovermelho (Parapiptadenia rigida), a canafístula (Peltophorum dubium), a timbaúva (Enterolobium contortisiliquum), o louro (Cordia trichotoma) e a canjerana (Cabralea canjerana), espécies emergentes que perdem suas folhas durante o inverno, caracterizando a floresta como estacional (REITZ et al., 1988).

Para Kray e Jarenkow (2003) as formações florestais, no Rio Grande do Sul, apresentam ampla variação na composição florística e na organização espacial. Essas variações devem-se principalmente a diferenças de altitude, determinadas pela presença do Planalto Sul-Brasileiro, e a proximidade ou distanciamento dos dois corredores de entrada de espécies tropicais no Estado: o Leste, entre as encostas da Serra Geral e Oceano Atlântico, e o Oeste, ao longo da bacia dos rios Paraná-Uruguai (RAMBO, 1961; JARENKOW e WAECHTER, 2001; KRAY e JARENKOW, 2003).

No Brasil, as Florestas Estacionais Deciduais ocorrem em manchas, desde a caatinga até o rio Uruguai (IBGE, 1995). Estas florestas são as mais ameaçadas (KHURANA e SINGH, 2001; FIGUEIREDO e SCARIOT, 2003) e as menos estudadas e conhecidas das formações florestais (WIKANDER, 1984; FIGUEIREDO e SCARIOT, 2003) das fitofisionomias tropicais, mas mesmo assim têm recebido pouca atenção em relação às Florestas Úmidas (MURPHY e LUGO, 1986; FIGUEIREDO e SCARIOT, 2003). Estas florestas são bastante visadas para a extração de madeiras, pois suas espécies mais abundantes têm elevado valor econômico (IBGE, 1995). Atualmente, essas se encontram altamente fragmentadas, com poucos estudos de longa duração, visando o manejo, uso e conservação desses recursos.

$\mathrm{O}$ uso de áreas demarcadas em levantamentos florísticos e fitossociológicos abre perspectivas para o desenvolvimento de pesquisas relacionadas à ecologia, à fenologia e à dinâmica das populações ali instaladas (WEISER e GODOY, 2001), e de sua estrutura florestal, a qual se baseia na organização dos vegetais em agrupamentos, por meio de análise florística e distribuição espacial das espécies (LONGHI, 1991) que, dentro das formações, apresentam grande diversidade.

Assim, o termo "formação" designa um tipo vegetacional definido, com um conjunto de formas de vida de ordem superior composto por uma fisionomia homogênea, possuindo uma estrutura complexa. Portanto, dentro da formação existem divisões de heterogeneidade estrutural que são definidas como subformações que se diferenciam por fácies específicas (IBGE, 1992).

Desta forma, técnicas multivariadas de agrupamento, visando o reconhecimento de diferenças dentro de formações florestais em fisionomias homogêneas por meio de grupos florísticos, auxiliam no conhecimento e determinação de grupos sucessionais e ecológicos.

Dentre as técnicas, o método objetivo (numérico) Twinspan (Two-Way INdicato SPecies ANalysis) é amplamente utilizado na análise de vegetação por possibilitar ordenar dicotomicamente, por meio de agrupamentos, as unidades amostrais e indicar as espécies codificantes determinantes desses grupos (FELFILI et al., 2007). Deste modo, o método numérico auxilia no conhecimento da floresta, caracterizando sua estrutura pelas subformações, possibilitando desta maneira, o planejamento de atividades de manejo diferenciadas para a recuperação, o uso e a conservação dos recursos florestais.

Assim o presente trabalho teve como objetivo principal descrever a fitossociologia de um fragmento florestal localizado no CESNORS (Centro de Educação Superior Norte-RS), com vistas a conhecer os grupos ecológicos e a presença de subformações fisionômicas e estruturais, com auxílio da composição florística, como também, determinar os diferentes estágios sucessionais da floresta secundária em estudo.

\section{MATERIAIS E MÉTODOS}

\section{Caracterização do local}

O fragmento estudado possui aproximadamente 60 ha da floresta estacional decidual submontana (LEITE e KLEIN, 1990;

Ci. Fl., v. 21, n. 3, jul.-set., 2011 
OLIVEIRA-FILHO et al., 2006). Está localizado no município de Frederico Westphalen, dentro das áreas do Centro de Educação Superior Norte - RS (CESNORS) e Colégio Agrícola de Frederico Westphalen (Figura 1). As coordenadas geográficas da área são $53^{\circ} 25^{\prime} 59.26^{\prime} \mathrm{O}$ e $27^{\circ} 23^{\prime} 44.40^{\prime \prime} \mathrm{S}$ e a altitude com variação entre 520 a $550 \mathrm{~m}$, apresentando relevo suaveondulado.

O clima regional, conforme a classificação de Köeppen, é do tipo Subtropical Temperado úmido (Cfa), caracterizado pela temperatura do mês mais quente superior a $22^{\circ} \mathrm{C}$, e a do mês mais frio superior a $3^{\circ} \mathrm{C}$, ao passo que as ocorrências médias de geadas ficam entre 5 a 15 por ano. A região apresenta um equilíbrio no regime pluviométrico pela ausência de estação seca definida, tendo uma média pluviométrica anual de $1900 \mathrm{~mm}$, com variação mensal em torno de $130-197 \mathrm{~mm}$ (MORENO, 1961; NIMER, 1990). Os solos predominantes na região são do tipo Latossolos Vermelhos Distroférricos pelo sistema brasileiro de classificação de solos (IBGE, 2002; EMBRAPA, 2006).

\section{Amostragem}

Foram demarcadas 10 parcelas permanentes de 20 x 50 m, com orientação do maior comprimento no sentido leste-oeste, distribuídas de forma aleatória, sendo cada uma delas constituída por 10 subparcelas quadradas de $100 \mathrm{~m}^{2}$, totalizando 1 ha de área amostral. Em cada parcela foram medidos todos os indivíduos arbóreos e arborescentes vivos e mortos em pé, como também, os cipós lenhosos, sendo considerados num único grupo amostral, com exceção da espécie, Seguieria aculeata que foi facilmente identificada pelo caule armado, com medição de todos os diâmetros na altura do peito $(\mathrm{DAP}) \geq 5 \mathrm{~cm}$.

Cada indivíduo incluído na parcela foi numerado com etiqueta de alumínio e teve sua altura total medida. Foram coletados materiais botânicos para identificação dos indivíduos que não foram determinados localmente. Para identificar os referidos materiais valeu-se das coleções do herbário do Departamento de Ciências Florestais da Universidade Federal de Santa Maria (HDCF/ UFSM), consulta à literatura e especialistas na área. Os materiais coletados foram incorporados ao acervo do HDCF e as duplicatas foram enviadas para a coleção didática do curso de Engenharia Florestal do CESNORS. As espécies foram classificadas nas famílias botânicas distinguidas pelo Angiosperm Phylogeny Group II (APG II, 2003).

\section{Análise de dados}

A análise da estrutura horizontal da floresta foi obtida pelo programa Fitoanálise 4.0, desenvolvido por CARVALHO JR., et al. (2000), referentes aos fitossociológicos de densidade, frequência e dominância absolutas e relativas; valores de importância e cobertura das espécies, além dos índices de diversidade de Shannon (H') e Pielou (J').

A distribuição da densidade dos indivíduos, por classes de diâmetro e altura, foi organizada em planilhas do Microsolf Excel, sendo os diâmetros empregados em classes crescentes e amplitudes que compensassem o decréscimo abrupto nas classes de maiores tamanhos.

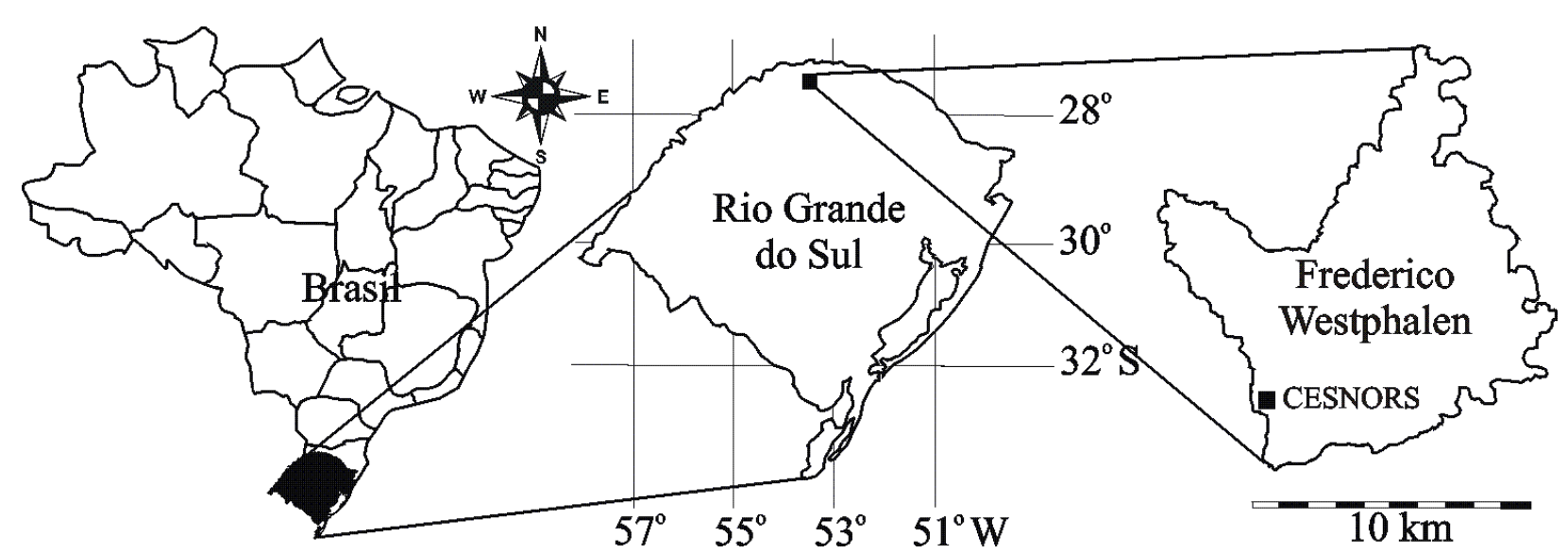

FIGURA 1: Localização da área de estudo (ロ), no município de Frederico Westphalen, RS.

FIGURE 1: Study location ( $\mathbf{\bullet})$, in Frederico Westphalen, RS state, Brazil. 
A análise de agrupamento foi pelo método hierárquico, divisível e politética, do método TWINSPAN, conforme Jongman et al., (1995) e Felfili et al. (2007). O programa computacional utilizado para análise foi o PC-ORD for Windows versão 5.0 (MCCUNE e MEFFORD, 2006), com a matriz de densidade de indivíduos de 79 espécies em 100 parcelas. As divisões dos grupos foram de acordo com o autovalor $\geq 0,30$, mas considerando somente aquelas que puderam ser explicadas e que apresentassem importância ecológica (ARAUJO et al., 2004; FELFILI et al., 2007; RIBEIRO et al., 2007). Os pontos de corte estipulados para as pseudoespécies foram $0,2,5,10$ e 20 , conforme Araujo et al. (2004).

\section{RESULTADOS E DISCUSSÃO}

\section{Estrutura fitossociológica e florística}

A amostragem da vegetação foi suficiente podendo ser constatada pela curva espécie-área (Figura 2). Sendo possível observar a linha de tendência do modelo logarítmico, com estabilização no acréscimo de novas espécies entre 7.000 e 8.000 $\mathrm{m}^{2}$ de área amostral e indicando forte relação entre área e espécie com $\mathrm{R}^{2} 0,98$.

No fragmento florestal amostrou-se um total de 1.461 indivíduos em um hectare, totalizando uma área basal de $38,5 \mathrm{~m}^{2} /$ ha para classe de DAP $\geq 5$ $\mathrm{cm}$. Entretanto, considerando apenas os indivíduos com $\mathrm{DAP} \geq 9,5 \mathrm{~cm}(\mathrm{CAP} \geq 30,0 \mathrm{~cm})$ o valor diminuiu para 660 ind.ha $^{-1}$, sendo o valor superior aos encontrados por Vaccaro e Longhi (1995), Hack et al. (2005), Scipioni (2008) em diferentes regiões de floresta estacional no Rio Grande do Sul.

A composição florística foi composta por 79 espécies distribuídas em 31 famílias e 69 gêneros. A família Fabaceae foi a que apresentou maior número de espécie, 14 no total, seguida de Myrtaceae (8), Meliaceae (6), Euphorbiaceae (5), Sapindaceae (5), Rutaceae (4) e Salicaceae (4), conforme Tabela 1. A elevada riqueza florística das famílias Fabaceae e Myrtaceae é característica das florestas estacionais do Rio Grande do Sul (LONGHI et al., 1999; HACK et al., 2005; SCIPIONI, 2008).

Entretanto, a florística das florestas do Alto Uruguai apresenta espécies com ocorrência dominante ou restrita fitogeograficamente em relação às demais florestas estacionais do Estado, que torna a florística arbórea distinta, podendo isso ser constatado em diversos trabalhos fitossocilógicos e florísticos regionais (LONGHI et al., 1999; JARENKOW e WAECHTER, 2001; HACK et al., 2005; LONGHI, et al., 2008; SCIPIONI, 2008; REITZ et al., 1988; SOBRAL e JARENKOW, 2006). Dentre as espécies contidas neste trabalho, característica dessa formação estacional, podese citar: Bauhinia forficata subespécie pruinosa, Balfourodendron riedelianum, Calliandra foliolosa, Eugenia burkartiana, Holocalyx balansae, Pilocarpus pennatifolius, Tetrorchidium rubrivenium, Diatenopteryx sorbifolia.

A riqueza florística encontrada neste estudo foi superior ao encontrado por VACCARO e LONGHI (1995), que encontraram 66 espécies, em estudo realizado na mesma região. No entanto,

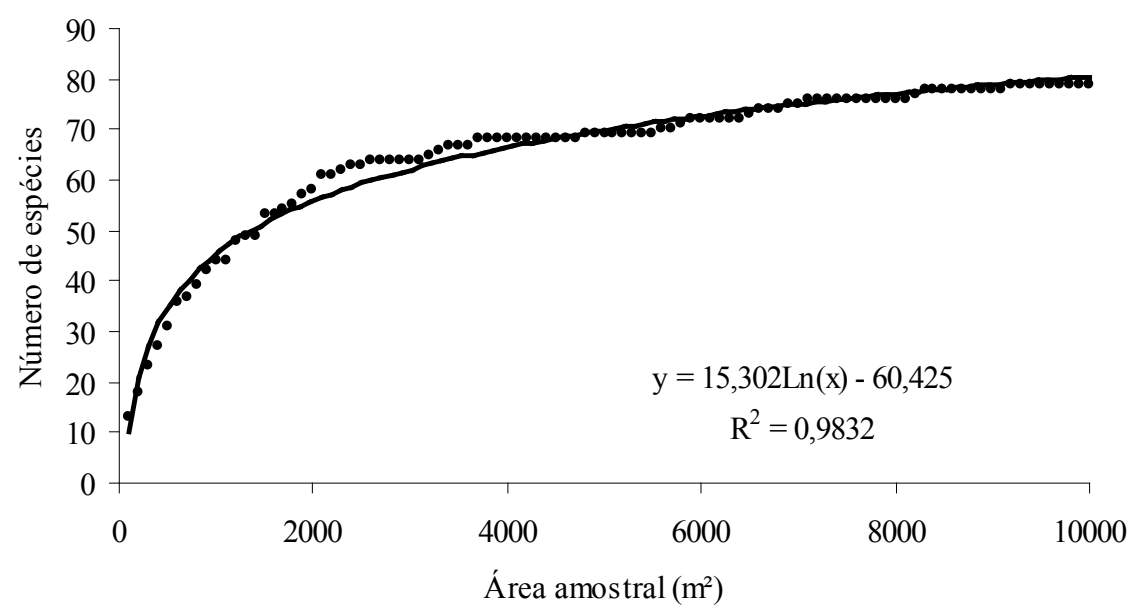

FIGURA 2: Curva espécie-área de um fragmento florestal em Frederico Westphalen, RS. FIGURE 2: Species-area curve of a forest patch in Frederico Westphalen, RS state.

Ci. Fl., v. 21, n. 3, jul.-set., 2011 
o seu limite de inclusão diamétrica foi maior que o presente estudo, proporcionando menor amostragem e consequentemente menor probabilidade de riqueza florística. Quanto ao índice de diversidade de Shannon (H') VACCARO e LONGHI (1995) foi pouco superior $\left(3,71\right.$ ind.nats $\left.^{-1}\right)$ ao encontrado neste trabalho, com um valor de 3,49 nats. indivíduo ${ }^{-1}$, isso se deve às distintas metodologias amostrais, dificultando comparações consistentes. Contudo, ambas refletem um alto índice de diversidade para a floresta estacional no Rio Grande do Sul. O índice de Equabilidade de Pielou encontrado foi de 0,79 $(\mathrm{J})$, sugerindo uma distribuição homogênea das espécies ao longo das unidades amostrais.

TABELA 1: Fitossociologia da floresta estacional decidual no município de Frederico Westphalen, RS. $\mathrm{DA}=$ Densidade absoluta $\left(\right.$ ind. ha $\left.{ }^{-1}\right) ; \mathrm{DoA}=$ Dominância absoluta $\left(\mathrm{m}^{2} \cdot \mathrm{ha}^{-1}\right) ; \mathrm{FA}=$ Frequência absoluta (\%);VI = Valor de importância (\%).

TABLE 1: Phytossociology of the seasonal deciduous forest in Frederico Westphalen, RS. DA=Absolute density $\left(\right.$ ind. ha $\left.\mathrm{a}^{-1}\right) ; \mathrm{DoA}=$ Absolute dominance $\left(\mathrm{m}^{2} \cdot \mathrm{ha}^{-1}\right) ; \mathrm{FA}=$ Absolute frequency $(\%) ; \mathrm{VI}=$ Importance value (\%).

\begin{tabular}{|c|c|c|c|c|c|}
\hline Família & Espécie & DA & DoA & FA & VI \\
\hline Euphorbiaceae & Gymnanthes concolor Spreng. & 240 & 1,29 & 73,00 & 28,16 \\
\hline Lauraceae & Nectandra megapotamica (Spreng.) Mez & 69 & 4,16 & 44,00 & 20,58 \\
\hline Meliaceae & Trichilia claussenii C.DC. & 130 & 1,84 & 57,00 & 20,22 \\
\hline Moraceae & Sorocea bonplandii (Baill.) W. C. Burger et al. & 88 & 0,67 & 44,00 & 12,82 \\
\hline Fabaceae & Holocalyx balansae Micheli & 10 & 3,55 & 9,00 & 10,95 \\
\hline Sapotaceae & Chrysophyllum marginatum (Hook. \& Arn.) Radlk. & 38 & 1,66 & 29,00 & 10,24 \\
\hline Myrtaceae & Eugenia schuechiana O. Berg & 62 & 0,20 & 32,00 & 8,43 \\
\hline Rutaceae & Pilocarpus pennatifolius Lem. & 50 & 0,29 & 36,00 & 8,29 \\
\hline Fabaceae & Apuleia leiocarpa (Vogel) J. F. Macbr. & 13 & 2,06 & 11,00 & 7,51 \\
\hline Meliaceae & Cabralea canjerana (Vell.) Mart. & 14 & 1,83 & 13,00 & 7,20 \\
\hline Sapindaceae & Diatenopteryx sorbifolia Radlk. & 12 & 1,90 & 11,00 & 7,01 \\
\hline Cyatheaceae & Cyathea atrovirens (Langsd. \& Fisch.) Domin & 70 & 0,46 & 6,00 & 6,67 \\
\hline Meliaceae & Trichilia catigua A.Juss. & 30 & 0,28 & 25,00 & 5,65 \\
\hline Fabaceae & Lonchocarpus sp. & 17 & 0,93 & 17,00 & 5,54 \\
\hline Sapindaceae & Cupania vernalis Cambess. & 29 & 0,71 & 12,00 & 5,22 \\
\hline Myrtaceae & Eugenia rostrifolia D. Legrand & 28 & 0,25 & 20,00 & 4,87 \\
\hline Fabaceae & Erythrina falcata Benth. & 7 & 1,35 & 7,00 & 4,80 \\
\hline Sapotaceae & Chrysophyllum gonocarpum (Mart. \& Eichler) Engl. & 20 & 0,54 & 17,00 & 4,72 \\
\hline Meliaceae & Cedrela fissilis Vell. & 9 & 1,12 & 9,00 & 4,57 \\
\hline Euphorbiaceae & Tetrorchidium rubrivenium Poepp. et Endl. & 15 & 0,87 & 9,00 & 4,32 \\
\hline Flacourtiaceae & Casearia sylvestris Sw. & 14 & 0,56 & 13,00 & 3,90 \\
\hline Rutaceae & Balfourodendron riedelianum (Engl.) Engl. & 17 & 0,41 & 14,00 & 3,84 \\
\hline Fabaceae & Machaerium stipitatum Vogel & 18 & 0,32 & 15,00 & 3,78 \\
\hline Lauraceae & Nectandra lanceolata Ness et Mart.ex Nees & 10 & 0,71 & 10,00 & 3,67 \\
\hline Sapindaceae & Allophylus edulis (A. St.-Hil. et al.) Radlk. & 13 & 0,50 & 11,00 & 3,44 \\
\hline Fabaceae & Calliandra foliolosa Benth. & 20 & 0,09 & 15,00 & 3,33 \\
\hline Fabaceae & Machaerium paraguariense Hassl. & 17 & 0,19 & 13,00 & 3,15 \\
\hline Araliaceae & Schefflera morototoni (Aubl.) Maguire et al. & 6 & 0,80 & 5,00 & 3,05 \\
\hline NI & Não identificada & 10 & 0,51 & 8,00 & 2,93 \\
\hline Fabaceae & Inga marginata Willd. & 14 & 0,09 & 12,00 & 2,57 \\
\hline Phytolaccaceae & Phytolacca dioica $\mathrm{L}$. & 1 & 0,88 & 1,00 & 2,48 \\
\hline Euphorbiaceae & Sebastiania brasiliensis Spreng. & 11 & 0,14 & 11,00 & 2,38 \\
\hline Euphorbiaceae & $\begin{array}{l}\text { Sebastiania commersoniana (Baill.) L. B. Sm. et } \\
\text { Downs }\end{array}$ & 7 & 0,41 & 6,00 & 2,23 \\
\hline Urticaceae & Urera baccifera (L.) Gaudich. & 15 & 0,09 & 8,00 & 2,18 \\
\hline Boraginaceae & Cordia trichotoma (Vell.) Arráb. ex Steud. & 4 & 0,53 & 4,00 & 2,10 \\
\hline
\end{tabular}


TABELA 1: Continuação ...

TABLE 1: Continued ...

\begin{tabular}{|c|c|c|c|c|c|}
\hline Família & Espécie & DA & DoA & FA & VI \\
\hline Fabaceae & Dalbergia frutescens (Vell.) Britton & 5 & 0,50 & 4,00 & 2,09 \\
\hline Achatocarpaceae & Achatocarpus praecox Griseb. & 9 & 0,26 & 5,00 & 1,85 \\
\hline Ulmaceae & Trema micrantha (L.) Blume & 16 & 0,10 & 4,00 & 1,82 \\
\hline Annonaceae & Rollinia salicifolia Schltdl. & 7 & 0,14 & 7,00 & 1,63 \\
\hline Solanaceae & Solanum sanctaecatharinae Dunal & 8 & 0,10 & 6,00 & 1,49 \\
\hline Myrtaceae & Campomanesia xanthocarpa O. Berg & 6 & 0,14 & 6,00 & 1,47 \\
\hline Meliaceae & Guarea macrophylla Vahl & 8 & 0,04 & 7,00 & 1,46 \\
\hline Fabaceae & Myrocarpus frondosus M. Allemão & 7 & 0,10 & 6,00 & 1,42 \\
\hline Meliaceae & Trichilia elegans A.Juss. & 8 & 0,02 & 7,00 & 1,40 \\
\hline Phytolaccaceae & Seguieria aculeata Jacq. (cipó) & 8 & 0,05 & 6,00 & 1,35 \\
\hline Lauraceae & Ocotea diospyrifolia (Meisn.) Mez & 6 & 0,07 & 6,00 & 1,29 \\
\hline Rubiaceae & Randia ferox (Cham. \& Schltdl.) DC. & 6 & 0,13 & 4,00 & 1,22 \\
\hline Loganiaceae & Strychnos brasiliensis (Spreng.) Mart. & 6 & 0,10 & 4,00 & 1,13 \\
\hline Styracaceae & Styrax leprosus Hook. et Arn. & 5 & 0,08 & 5,00 & 1,13 \\
\hline Myrtaceae & Eugenia involucrata DC. & 5 & 0,05 & 5,00 & 1,06 \\
\hline Rosaceae & Prunus myrtifolia (L.) Urb. & 4 & 0,09 & 4,00 & 0,96 \\
\hline Flacourtiaceae & Casearia decandra Jacq. & 4 & 0,07 & 4,00 & 0,93 \\
\hline Boraginaceae & Cordia americana (L.) Gottschling \& J.E.Mill. & 2 & 0,22 & 2,00 & 0,93 \\
\hline Ulmaceae & Celtis iguananea (Jacq.) Sarg. & 5 & 0,06 & 3,00 & 0,84 \\
\hline Bignoniaceae & Jacaranda micrantha Cham. & 3 & 0,11 & 3,00 & 0,84 \\
\hline Monimiaceae & Hennecartia omphalandra J. Poiss. & 4 & 0,03 & 4,00 & 0,82 \\
\hline Fabaceae & $\begin{array}{l}\text { Bauhinia forficata Link subsp. pruinosa Fortunato \& } \\
\text { Wunderlin (Vogel) }\end{array}$ & 4 & 0,03 & 4,00 & 0,81 \\
\hline Fabaceae & Bauhinia forficata Link & 4 & 0,02 & 4,00 & 0,79 \\
\hline Bombacaceae & Ceiba speciosa (A. St-Hil.) Ravenna & 2 & 0,15 & 2,00 & 0,75 \\
\hline Flacourtiaceae & Banara tomentosa Clos & 3 & 0,07 & 3,00 & 0,73 \\
\hline Myrtaceae & Campomanesia guazumifolia (Cambess.) O. Berg & 3 & 0,06 & 3,00 & 0,70 \\
\hline Sapindaceae & Matayba elaeagnoides Radlk. & 3 & 0,09 & 2,00 & 0,68 \\
\hline Myrtaceae & Plinia rivularis (Cambess.) Rotman & 1 & 0,18 & 1,00 & 0,65 \\
\hline Nyctaginaceae & Pisonia zapallo Griseb. & 3 & 0,08 & 2,00 & 0,65 \\
\hline Rutaceae & Zanthoxylum rhoifolium L. & 3 & 0,01 & 3,00 & 0,58 \\
\hline Arecaceae & Syagrus romanzoffiana (Cham.) Glassman & 3 & 0,05 & 2,00 & 0,57 \\
\hline Simaroubaceae & Picrasma crenata (Vell.) Engl. & 2 & 0,04 & 2,00 & 0,47 \\
\hline Rutaceae & Zanthoxylum petiolare A. St.-Hil. \& Tul. & 2 & 0,02 & 2,00 & 0,42 \\
\hline Euphorbiaceae & Alchornea triplinervia (Spreng.) Müll. Arg. & 2 & 0,01 & 2,00 & 0,40 \\
\hline Fabaceae & Parapiptadenia rigida (Benth.) Brenan & 2 & 0,01 & 2,00 & 0,39 \\
\hline Myrtaceae & Eugenia burkartiana (D. Legrand) D. Legrand & 2 & 0,01 & 2,00 & 0,39 \\
\hline Flacourtiaceae & Xylosma pseudosalzmannii Sleumer & 2 & 0,01 & 2,00 & 0,38 \\
\hline Boraginaceae & Cordia ecalyculata Vell. & 1 & 0,07 & 1,00 & 0,37 \\
\hline Malvaceae & Luehea divaricata Mart. \& Zucc. & 1 & 0,03 & 1,00 & 0,27 \\
\hline Araliaceae & Aralia warmingiana (Marchal) J. Wen & 1 & 0,02 & 1,00 & 0,24 \\
\hline Hippocrateaceae & Pristimera andina Miers & 1 & 0,01 & 1,00 & 0,22 \\
\hline Fabaceae & Albizia niopoides (Spruce ex Benth.) Burkart & 1 & 0,01 & 1,00 & 0,20 \\
\hline Myrtaceae & Myrcianthes pungens (O. Berg) D. Legrand & 1 & 0,01 & 1,00 & 0,20 \\
\hline Styracaceae & Styrax acuminatus Pohl & 1 & 0,00 & 1,00 & 0,19 \\
\hline Sapindaceae & Allophylus guaraniticus (A. St-Hil.) Radlk. & 1 & 0,00 & 1,00 & 0,19 \\
\hline Cipó & Cipós & 102 & 0,66 & 59,00 & 15,45 \\
\hline Morta & Árvore morta em pé & 50 & 2,18 & 38,00 & 13,44 \\
\hline Total & & 1.461 & 38,50 & 872,00 & 300,00 \\
\hline
\end{tabular}

Ci. Fl., v. 21, n. 3, jul.-set., 2011 
Na figura 3, encontra-se a distribuição dos indivíduos em classes diamétrica e altura. A distribuição diamétrica apresentou variação de 5 a $106 \mathrm{~cm}$ de diâmetro, com destaque para Phytolacca dioica $(106 \mathrm{~cm})$ e Holocalyx balansae $(104 \mathrm{~cm})$ com os maiores diâmetros amostrados, sendo que a menor classe entre 5 e $10 \mathrm{~cm}$ representou mais da metade dos indivíduos amostrados $(58,2 \%)$, contribuindo assim, para tendência normal da curva em "J" invertido. Nas classes de altura no intervalo entre 5 e $10 \mathrm{~m}$, foi verificada a maior concentração dos indivíduos, representando metade dos indivíduos amostrados nessa classe $(50,0 \%)$ e as alturas máximas ultrapassaram $30 \mathrm{~m}$.

As três espécies arbóreas que apresentaram maior densidade no fragmento (Tabela 1) foram: Gymnanthes concolor (240 ind. ha $\left.{ }^{-1}\right)$, Trichilia claussenii (130 ind. ha ${ }^{-1}$ ) Sorocea bonplandii (88ind. $\mathrm{ha}^{-1}$ ), as quais são dominantes dos estratos inferiores da floresta e representaram $36,1 \%$ dos indivíduos amostrados. As espécies Cyathea atrovirens (xaxim), pertencente à divisão Pteridophyta, com 70 ind. ha ${ }^{-1}$, e Nectandra megapotamica (69 ind. ha ${ }^{-1}$ ) destacaram-se, também, pela sua alta densidade.

As árvores mortas em pé e lianas apresentaram $50(3,4 \%)$ e $108(7,5 \%)$ indivíduos amostrados por hectare respectivamente, sendo esse valor percentual de árvores mortas em pé foi baixo em relação ao outros trabalhos que detectaram valores superiores a 5\% (LONGHI et al, 1999; LONGHI et al., 2008). Assim, a floresta encontra-se em estágio médio a avançada de sucessão, principalmente pela presença e número expressivo de cipós na área, conforme resolução Conama número 06 de 1994. Contudo, essa resolução está especificada para a
Floresta Ombrófila Densa e Semidecidual no Estado do Rio de Janeiro, sendo que novas resoluções específicas devem ser elaboradas para os diferentes subgrupos de formação fitogeográfico do IBGE (1992), pois cada subgrupo apresenta características estruturais, fisionômicas e florísticas distintas nos diferentes níveis sucessionais.

No fragmento florestal estudado, as espécies com os maiores índices de valor de importância (VI), foram Gymnanthes concolor (28,2), Nectandra megapotamica $(20,6)$, Trichilia claussenii $(20,2)$, Chrysophyllum marginatum (10,2), Eugenia schuechiana $(8,4), \quad$ Pilocarpus pennatifolius $(8,3)$, Apuleia leiocarpa $(7,5)$ e Cabralea canjerana $(7,2)$, conforme Tabelas 1 , onde as espécies foram ordenadas de forma decrescente pelo maior VI.

Dentre essas espécies, o alto VI de Apuleia leiocarpa e Cabralea canjerana está correlacionado ao alto valor de área basal, sendo árvores dominantes dos estratos superiores. Logo, Nectandra megapotamica e Chrysophyllum marginatum apresentaram frequência, área basal e densidade média que contribuíram para sua valoração na floresta, sendo ambas as espécies características do estrato médio. As demais espécies típicas do estrato inferior de pequenas dimensões apresentaram elevada importância principalmente em decorrência dos altos pesos de densidade e frequência, isso também pode ser considerado para as lianas $(15,4)$ na quinta posição de VI. As árvores mortas em pé $(13,4)$ ocupam a quarta posição no VI, sendo esse componente importante na constituição da floresta, possuindo variação diamétrica entre 5 e $60 \mathrm{~cm}$ e valor médio de $18,8 \mathrm{~cm}$.
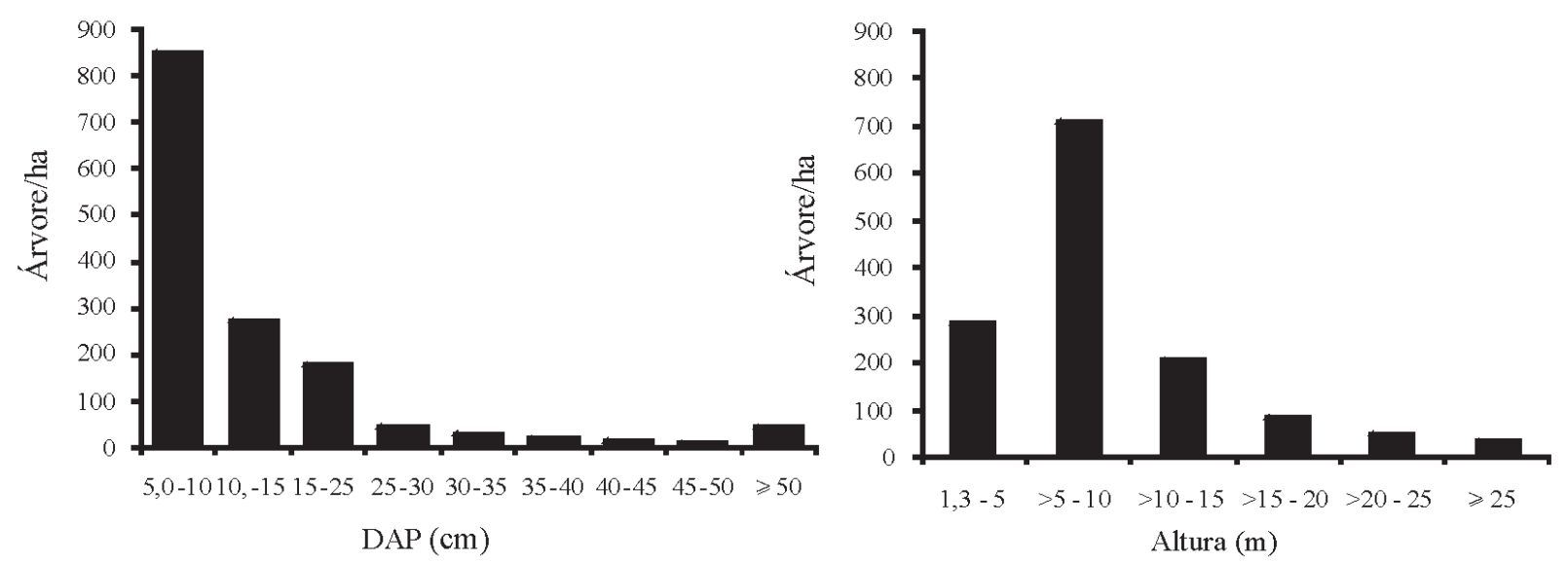

FIGURA 3: Distribuição da densidade dos indivíduos por hectare nas classes de diâmetro e altura. FIGURE 3: Density distribution of individuals by hectare in classifications of diameter and height. 


\section{Caracterização das subformações}

A presença de subformações no interior do fragmento foi relevante, resultando na primeira divisão na análise de grupos florísticos, com autovalor de 0,3571. Esse valor foi relevante, sendo superior 0,30 o que caracteriza variação significativa dos dados (FELFILI et al., 2007) para separação das unidades amostrais em grupos distintos, os quais refletem diferentes condições na composição florística no fragmento em decorrência da distribuição espacial das espécies.

Entretanto, em decorrência da uniformidade da geologia e geomorfologia da área e, logo, não abrangendo um gradiente significativo com variação ambiental de solos e hidromorfía, foi constatado o predomínio de um grande grupo que abrangeu $87 \%$ das unidades amostrais (Subformação 1), caracterizando fisionomicamente o fragmento numa floresta homogênea em fase secundária avançada.

$\mathrm{O}$ aspecto que caracteriza o estágio sucessional secundário da Subformação1 foram as espécies indicadoras resultantes, Trichilia claussenii e Gymanthes concolor, que são predominantemente dominantes das posições verticais inferiores da floresta em dossel denso, caracterizando a sucessão florestal secundária avançada (SCIPIONI, 2008). Vaccaro et al. (1999) enfatiza que essas são exclusivas do sub-bosque em condição sucessional de floresta madura e secundária, em estudo realizado numa floresta estacional no nordeste do RS, refletindo da mesma forma, para formação estudada no noroeste do Estado, estando essa estrutura florestal representada pela Figura 4.

No outro grupo da primeira divisão, com 13 unidades amostrais, apresentou uma nova divisão (3), com autovalor significativo de 0,6529, dando origem a subformação 2 , fisionomicamente distinguível pela presença de fetos arborescentes (xaxins) no estrato inferior da floresta. $\mathrm{Na}$ subformação 3 que não apresentou espécie indicadora e nem Xaxins, teve uma fisionomia semelhante à subformação 1. Entretanto, com diferentes espécies no dossel inferior, como, Sorocea bonplandii, Celtis iguananea e Cupania vernalis, caracterizando um estágio secundário inicial ocasionado por perturbações recentes no interior do fragmento, devido à queda natural de árvores ou processos de cortes seletivos.

As espécies indicadoras da subformação 2 foram Cyathea atrovirens e Tetrorchidium rubrivenium, sendo que Trichipteris atrovires uma espécie arborescente (xaxim) que apresentou

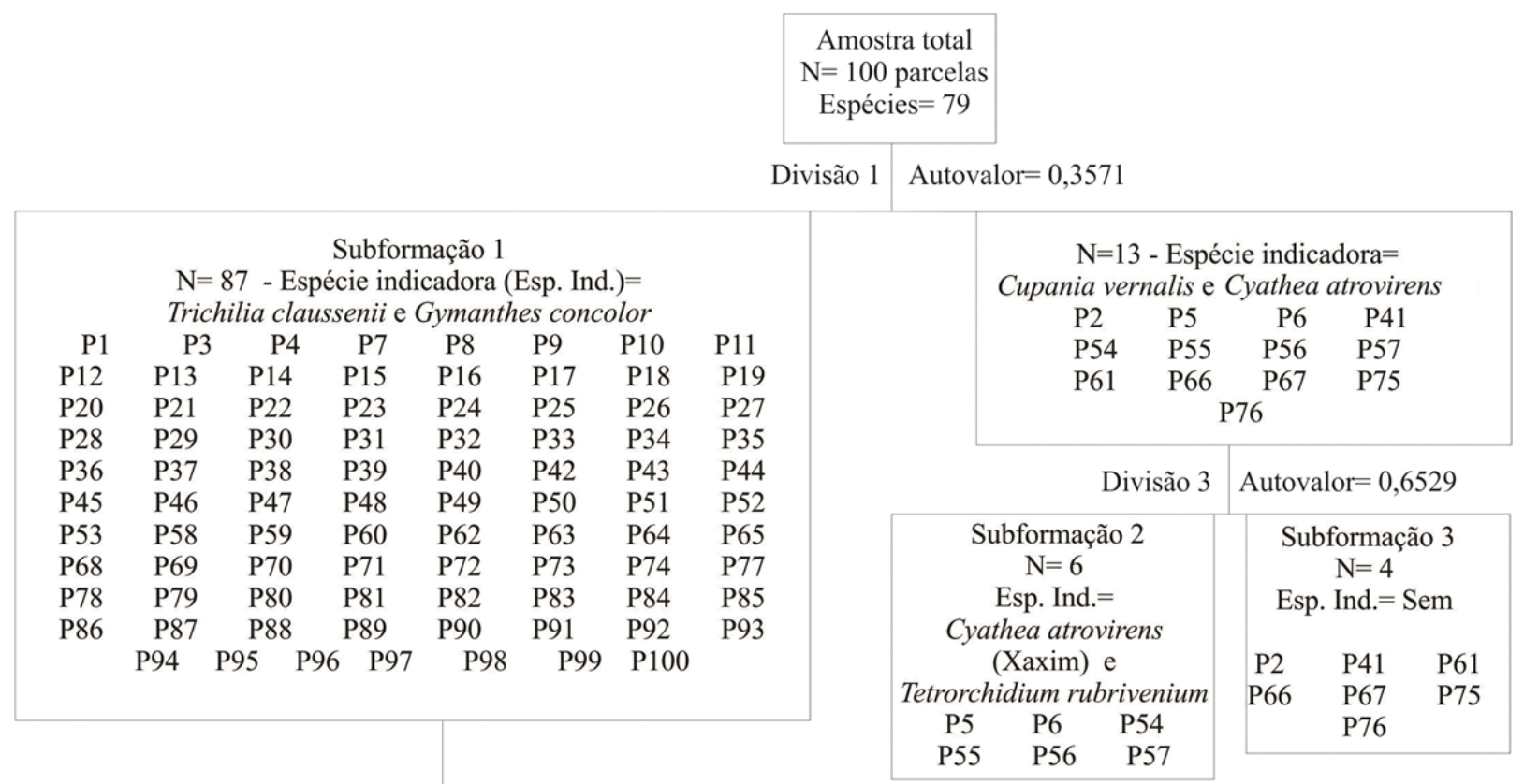

Divisão 2 Autovalor $=0,2652$

FIGURA 4: Dendrograma de agrupamento das subformações no fragmento florestal, Frederico Westphalen, RS.

FIGURE 4: Cluster dendrogram of sub-formations in the forest patch, Frederico Westphalen, RS state, Brazil. 
distribuição agregada com alta densidade de indivíduos nas parcelas de ocorrência (Figura 5). A média de indivíduos por unidade amostral de $100 \mathrm{~m}^{2}$ foi $13,2( \pm 3,63)$, com variação de 9 a 17 indivíduos por unidade amostral.

Esses valores de densidade foram inferiores ao encontrado por Schmitt e Windisch (2005) no Rio Grande do Sul para a espécie Alsophila setosa, outra espécie arborescente, que também apresentou o comportamento significativamente agrupado, com valores de $17,42( \pm 11,54)$ e $23,50( \pm 10,81)$ indivíduos por $100 \mathrm{~m}^{2}$, em duas áreas distintas. Mesmo assim, a densidade foi elevada para a distinção da subformação 2 em relação às demais, na análise de agrupamento. Giehl et al. (2007) estudando a distribuição espacial de espécies arbóreas em Santa Maria (RS), também constatou o mesmo comportamento para Alsophila setosa, que formou um único agrupamento na área de estudo, sem direcionalidade, sendo que as demais espécies formaram diversos grupos, com densidade e arranjo espacial variável.

Deste modo, o comportamento fitossociológico agrupado de espécies pteridófitas arborescentes está correlacionado com o fato da reprodução vegetativa. Devido ao estabelecimento de esporófitos jovens a partir de gametófitos em nichos com condições adequadas próximo da planta mãe, associado também com a propagação por meio de ramificações estoloníferas (MOREIRA, 2005; SCHMITT e WINDISCH, 2005). Tornando assim, a subformação 2 um grupo com estrutura e composição florística distinta no fragmento, com restrições ao uso em decorrência da fragilidade mecânica dos fetos arborescentes e a falta de estudos quanto a sua conservação e manejo.

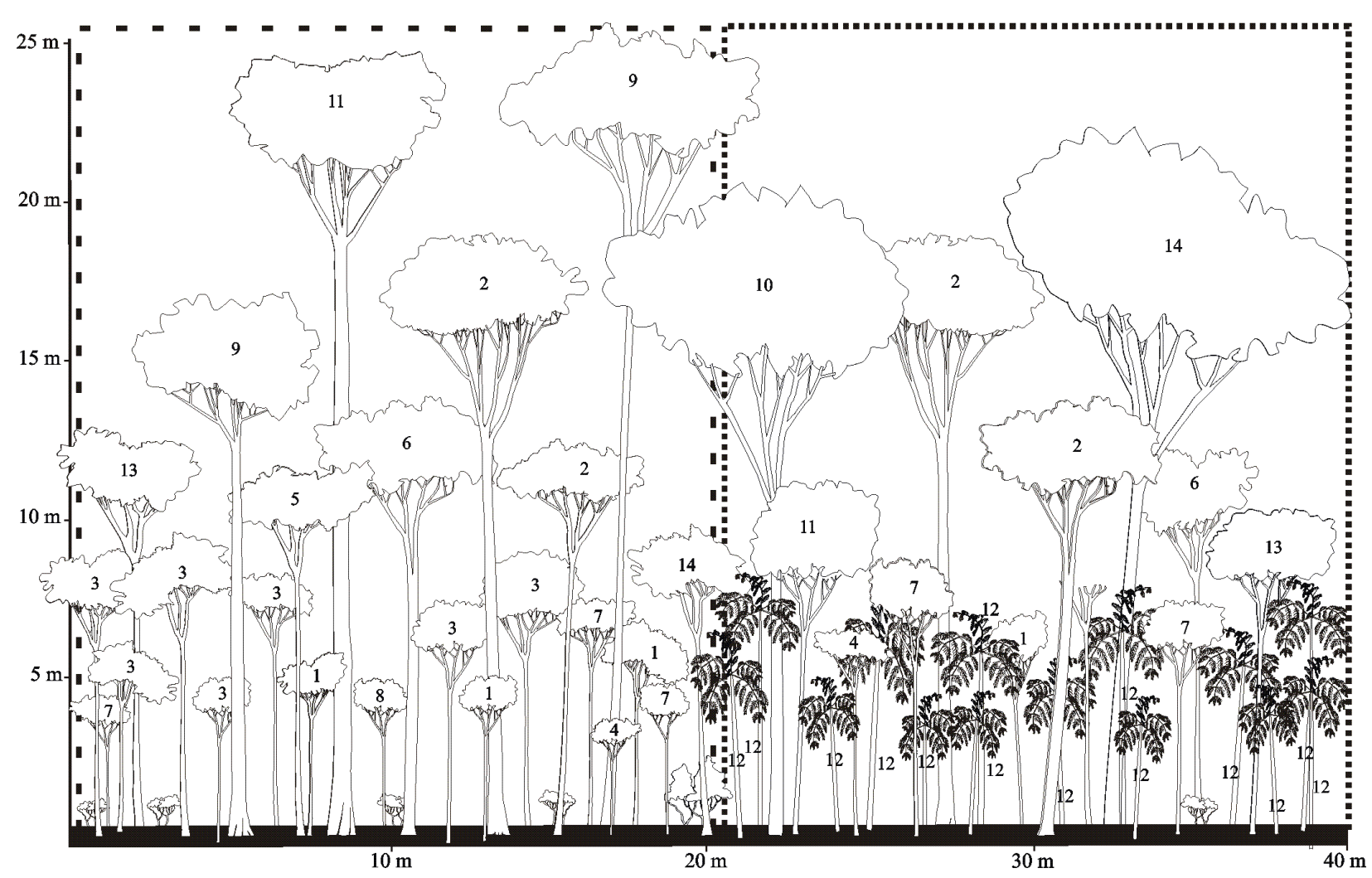

Espécies/ Species: 1 Gymnanthes concolor, 2 Nectandra megapotamica, 3 Trichilia claussenii, 4 Sorocea bonplandii, 5 Holocalyx balansae, 6 Chrysophyllum marginatum, 7 Eugenia schuechiana, 8 Pilocarpus pennatifolius, 9 Apuleia leiocarpa, 10 Cabralea canjerana, 11 Diatenopteryx sorbifolia, 12 Cyathea atrovirens (Pteridophyta-Xaxim), 13 Cupania vernalis, 14 Tetrorchidium rubrivenium.

FIGURA 5: Fitofisionomia das subformações $1(--)$ e $2(\cdots)$ no fragmento florestal, na Floresta Estacional Decidual em Frederico Westphalen, RS.

FIGURE 5: Forest physiognomy of sub-formations $1(--)$ and $2(\cdots)$ in a patch of seasonal deciduous forest in Frederico Westphalen, RS state, Brazil. 


\section{CONCLUSÕES}

No levantamento fitossociológico constatou-se que o fragmento florestal apresentou alta diversidade de espécies e grande variação na estrutura vertical e horizontal, em comparação com os diversos estudos existentes em floresta estacional no estado do Rio Grande do Sul.

O remanescente tem grande importância para estudos ecológicos de longa duração, além de apresentar espécies ameaçadas e de reconhecida importância comercial (Apuleia leiocarpa e Cabralea canjerana) entre as espécies de alto valor de importância.

O método de agrupamento do Twinspan permite identificar e diferenciar as subformações dentro do fragmento florestal em condições homogêneas de solos e relevo, formando grupos com espécies indicadoras dominantes, que através do conhecimento da autoecologia, distribuição sociológica vertical e mecanismos de reprodução das espécies, possibilita caracterizar os estágios sucessionais das florestas secundárias estacionais.

As espécies de maior densidade do dossel inferior e do sub-bosque foram as responsáveis pela diferenciação das subformações, condição sucessional e no aspecto fisionômico interno da floresta secundária. Assim, essa análise possibilita conhecer e fornecer parâmetros fisionômicos, para diferenciar e demarcar o espaço territorial, possibilitando assim, um planejamento distinto nas atividades de manejo e uso da floresta.

\section{AGRADECIMENTOS}

Aos pesquisadores que auxiliaram na identificação das espécies: José Newton Cardoso Marchiori (UFSM), Marcos Sobral (UFMG), Osmar dos Santos Ribas (MBM) e Solon Jonas Longhi (UFSM).

\section{REFERÊNCIAS BIBLIOGRÁFICAS}

APG II. An update of the Angiosperm Phylogeny Group classification for the ordens and families of flowering plants: APG II. Botanical Journal of the Linnaean Society, London, n. 141, p. 399-436, 2003.

ARAUJO, M. M. et al., Análise de agrupamento da vegetação de um fragmento de floresta estacional decidual aluvial, Cachoeira do Sul, RS, Brasil. Ciência Florestal, Santa Maria, v. 14, n. 1, p. 133-
147, 2004.

CARVALHO JR., L. A. et al. Fitoanálise: Versão 4.0 - 10. Santa Maria: 2000. Software para análise fitossociológica da vegetação.

CONAMA. Conselho Nacional de Meio Ambiente. Estabelece definições e parâmetros mensuráveis para análise de sucessão ecológica da Mata Atlântica no Rio de Janeiro. Resolução 006 de 1994.

EMBRAPA. Empresa Brasileira de Pesquisa Agropecuária, Centro Nacional de Pesquisa de Solos. Sistema de Classificação Brasileiro de Classificação de Solos. 2. ed. Rio de Janeiro: 2006. $306 \mathrm{p}$.

FELFILI, J. M. et al. Análise multivariada em estudos de vegetação. Brasília: Universidade de Brasília, Departamento de Eng. Florestal, 2007. 60 p. (Comunicações Técnicas Florestais, v.9, n.1).

FIGUEIREDO, I. B.; SCARIOT, A. Padrões de Polinização e Dispersão de Sementes de Espécies Arbóreas de Floresta Estacional Decidual, Brasil Central, 2003. In: CONGRESSO DE ECOLOGIA DO BRASIL, 6., 2003, Fortaleza. Anais... Fortaleza: Sociedade de Ecologia do Brasil, 2003. In: Cap. III Florestas Estacionais p. 439-441.

GIEHL, E. L. H. et al. Distribuição espacial de espécies arbóreas em uma floresta estacional em Santa Maria, sul do Brasil. Pesquisas: Botânica, São Leopoldo, n. 58, p. 215-226, 2007.

HACK, C. et al. Análise fitossociológica de um fragmento de floresta estacional decidual no município de Jaguari, RS. Ciência Rural, Santa Maria, v. 35, n.5, p. 1083-1091, set./out. 2005.

IBGE. Manual técnico da vegetação brasileira. Rio de Janeiro, 1992. 92 p.

IBGE. Zoneamento geoambiental e agroecológico do estado de Goiás: região noroeste. IBGE/Divisão de Geociências do Centro-Oeste, Rio de Janeiro, RJ, 1995.

IBGE. Mapa exploratório de solos do Rio Grande do Sul. Rio de Janeiro: Instituto Brasileiro de Geografia e Estatística, 2002. 1 mapa, digital, 120 $\mathrm{cm}$. Escala 1:100.000.

JARENKOW, J. A.; WAECHTER, J. L. Composição, estrutura e relações florísticas do componente arbóreo de uma floresta estacional no Rio Grande do Sul. Rev. bras. Bot., São Paulo, v. 24, n. 3, p. 263-272, 2001.

JONGMAN, R. H. G. et al. Data analysis in community and landscape ecology. 2 ed. Cambrigde: Cambrigde University Press, 1995. 299 p. 
KHURANA, E.; SINGH, S. J. Ecology of seed and Seedling Growth for Conservation and Restoration of Tropical Dry Forest: a review. Environmental Conservation, v. 28, p. 39-52, 2001.

KRAY, J. G.; JARENKOW, J. A. Estrutura do componente arbóreo de mata estacional de encosta do Parque de Itapuã, Viamão, RS, 2003. In: CONGRESSO DE ECOLOGIA DO BRASIL, 6., 2003, Fortaleza. Anais... Fortaleza: Sociedade de Ecologia do Brasil, 2003. Cap. III - Florestas Estacionais p. 452-453.

LEITE, P. F.; KLEIN, R. M. Fundação Instituto Brasileiro de Geografia e Estatística - IBGE. Geografia do Brasil - Região Sul. Rio de Janeiro: IBGE, 1990. p.113 - 150.

LONGHI, S. J. Aspectos fitossociológicos dos "Capões" na região de Carovi e Tupantuba, em Santiago, RS. Ciência Florestal, Santa Maria, v. 1, n. 1, p. 22-39, 1991.

LONGHI, S. J. et al. Composição florística e estrutura da comunidade arbórea de um fragmento florestal no município de Santa Maria, Brasil. Ciência Florestal, Santa Maria, v. 9, n. 1, p. 115133, 1999.

LONGHI, S. J. et al. Caracterização fitossociológica do estrato arbóreo em um remanescente de floresta estacional semidecidual, em Montenegro, RS. Ciência Rural, Santa Maria, v. 38, n. 6, p. 16301638, set. 2008.

MCCUNE, B.; MEFFORD, M. J. PC-ORD version 5.0. Multivariate analysis of ecological data. $\mathrm{MjM}$ Solfware Desing, Glaneden Beach, 2006.

MOREIRA, P. A. B. Efeito da criopreservação de esporos em nitrogênio líquido no desenvolvimento de gametófitos de Dicksonia sellowiana Hook. 2005. 64 f. Dissertação (Mestrado em Biologia Vegetal)-Universidade Federal de Santa Catarina, Florianópolis, 2005.

MOREnO, J. A. Clima do Rio Grande do Sul. Porto Alegre: Secretaria da Agricultura do Rio Grande do Sul, 1961. 42 p.

MURPHY, P. G.; LUGO, A. E. Ecology of Tropical Dry Forest. Annual Review of Ecology and Systematics, v. 17, p. 67-88, 1986.

NIMER, E. Fundação Instituto Brasileiro de Geografia e Estatística - IBGE. Geografia do Brasil - Região Sul. Rio de Janeiro: IBGE, 1990. p.151 - 187 .

OLIVEIRA-FILHO, A. T.; JARENKOW, J. A.; RODAL, M. J. N. Floristic relationships of seasonally dry forests of eastern South America based on tree species distribution patterns. In: PENNINGTON, R. T.; LEWIS, G. P.; RATTER, J. A. (Org.). Neotropical savannas and dry forests: Plant diversity, biogeography and conservation. Boca Raton: CRC Press, 2006, v.1, p. 159-192.

RAMBO, B. Migration routes of the south brasilian rain florest. Pesquisa botânica, São Leopoldo, v. 12, p. 1-54, 1961.

REITZ, R. et al. Projeto Madeira do Rio Grande do Sul. Porto Alegre: SUDESUL-GERS-IBDF, 1988.

RIBEIRO, S. B. et al. Diversidade e Classificação da comunidade arbórea da Floresta Ombrófila Mista da FLONA de São Francisco de Paula, RS. Ciência Florestal, Santa Maria, v. 17, n. 2, p. 101-108, abr./ jun. 2007.

SCHMITT, J. L.; WINDISCH, P. G. Aspectos ecológicos de Alsophila setosa Kaulf. (Cyatheaceae, Pteridophyta) no Rio Grande do Sul, Brasil. Acta bot. bras. v.19, n. 4, p. 859-865, 2005.

SCIPIONI, M. C. Análise dos padrões florísticos e estruturais da comunidade arbórea-arbustiva e da regeneração natural em gradientes ambientais na floresta estacional, RS, Brasil. 2008. 89 f. Dissertação (Mestrado em Engenharia Florestal)Universidade Federal de Santa Maria, 2008.

SOBRAL, M.; JARENKOW, J. A. (Org.). Flora arbórea e arborescente do Rio Grande do Sul, Brasil. São Carlos: Rima; Porto Alegre: Novo Ambiente, 2006. 350 p.

VACCARO, S.; LONGHI, S. J. Análise fitossociológica de algumas áreas remanescentes da Floresta do Alto Uruguai, entre os rios Ijuí e Turvo, no Rio Grande do Sul. Ciência Florestal, Santa Maria, v. 5, n. 1, p. 33-53, 1995.

VACCARO, S. et al. Aspectos da composição florística e categorias sucessionais do estrato arbóreo de três subseres de uma floresta estacional decidual, no município de Santa Tereza - RS. Ciência Florestal, Santa Maria, v. 9, n. 1, p. 1-18, 1999.

WEISER, V. L.; GODOY, S. A. P. Florística em um hectare de cerrado strictu sensu na arie-cerrado péde-gigante, Santa Rita do Passa Quatro, SP. Acta bot. Bras., São Paulo, v. 15, n. 2, p. 201-212, 2001. WIKANDER, T. Mechanismos de Dispersion de Diásporas de uma Selva Decídua em Venezuela. Biotropica, v.16, p. 276-285, 1984. 